\title{
Unified early and late Universe cosmology through dissipative effects in steep quintessential inflation potential models
}

\author{
Gustavo B. F. Lima ${ }^{1}$ and Rudnei O. Ramos ${ }^{2}$ \\ ${ }^{1}$ Observatório Nacional, 20921-400 Rio de Janeiro, RJ, Brazil \\ ${ }^{2}$ Departamento de Fisica Teorica, Universidade do Estado do Rio de Janeiro, 20550-013 Rio de Janeiro, RJ, Brazil
}

\begin{abstract}
By making use of a class of a steep exponential type of potentials, which has been recently used to describe quintessential inflation, we show how a unified picture for inflation, dark energy, and dark matter can emerge entirely through dissipative effects. Dissipation provides a way to extend the applicability of a larger class of these potentials in the sense of leading to a consistent early Universe inflationary picture and producing observables in agreement with the Planck legacy data. Likewise, dissipative effects lead to dark matter production with consistent abundances and, toward the recent time of the Universe, drives the potential energy of the scalar quintessential field to dominate again, essentially mimicking a cosmological constant by today, with all cosmological parameters consistent with the observations. Both early and late Universes are connected and have no kination period in between.
\end{abstract}

\section{INTRODUCTION}

By now, we have accumulated a large number of cosmological data coming from different sources and at increasing precision, as the ones obtained by the most recent cosmological probes, including data from the cosmic microwave background (CMB) anisotropies [1, galaxy clustering, e.g., baryon acoustic oscillations (BAO) 2,4, gravitational lensing [5], the large-scale structure (LSS) of the Universe [6] and supernovae [7. Putting all together, the observational data point to a Universe that fits the so-called concordance model of cosmology, or $\Lambda \mathrm{CDM}$ model, quite well. The $\Lambda \mathrm{CDM}$ model is the simplest cosmological model fitting existing data and describes our Universe in terms of a cosmological constant $\Lambda$ modeling dark energy for the recent accelerating expansion and cold dark matter (CDM), as the dominant energy components. Finally, the $\Lambda \mathrm{CDM}$ model is complemented by the idea of inflation as the paradigm to the solution of the big bang cosmological model for the early Universe.

Though inflation, dark energy and dark matter are considered as separated entities in general in the literature, there are proposals formulating different unified pictures for them $[8-13$. This is motivated by trying to have a simple picture for these different forms of energy that have prevailed in the early Universe (inflation) and in the recent cosmological history (dark energy and dark matter). In fact, the $\Lambda$ CDM model itself motivates these studies, remembering that the model itself faces unsolved theoretical issues, like the fine-tuning problem [14], the cosmic coincidence problem 15 and the unexplained numerical value of the cosmological constant itself.

In the present work, we propose a model implementing a typical quintessential inflation behavior, i.e., through a scalar field model that can play both the roles of the inflaton and the dark energy (in the form of a dynamical scalar-quintessence field), in the early and late cosmology histories of the Universe, respectively. Quintessential inflation models are abundant in the literature [16 25].
One of the main novelties in the model to be presented here is the role that dissipation will be making throughout the dynamics, from the very early inflationary Universe until the recent dark energy accelerated epoch. In addition, dissipation itself will serve us in producing the appropriate abundance of dark matter, thus naturally and nicely connecting the end of inflation with the big bang early radiation dominated Universe, passing by the matter-dominated regime and ending in the recent (dark energy-dominated) acceleration regime. We will realize this program in the context of the warm inflation picture [26] (see also Refs. [27, 28, for reviews), which also serves to motivate how energy can be exchanged in between the dark sector components (i.e., between the quintessential inflaton field and the dark matter).

Recently, quintessential inflation models motivated from warm inflation have been suggested [29, 30. These models have a number of attractive features. For example, one does not need to rely on gravitational particle production in these models as the leading mechanism for generating the radiation-dominated regime after inflation 1 like in the original quintessential inflation model by Peebles and Vilenkin [16. Besides, on a more fundamental level, there has recently been increased interest in how present field theory models describing either inflation, or dark energy, or both, can be accommodated in a consistent ultraviolet completion in quantum gravity/string theory. This gave origin to the recent so-called swampland conjectures (see, e.g., Ref. 39] for a thorough recent review and references therein), which puts in check the $\Lambda \mathrm{CDM}$ model, dark energy models and inflation models alike. In this perspective, warm inflation has been discussed as a possible and natural way to evade the issues brought about by these swampland conjectures [40 42].

\footnotetext{
1 Note, however, that there are other ways of reheating the Universe in quintessential inflation and that do not rely on gravitational particle production, like instant preheating [31, 32], curvaton reheating [33 35], Ricci reheating [36, 37] and reheating with a trap 38 .
} 
While the authors in Refs. [29, 30] formulated the quintessential inflation model in the warm inflation context with the Peebles and Vilenkin type of potential [16, here, instead, we will make use of a class of steep exponential potentials for quintessential inflation [43 47]. Contrary to the thawing type of models for quintessence, this is a freezing type of model (for a distinction between thawing and freezing models, see, e.g., Ref. [48). While thawing models are very sensitive to the initial conditions, the freezing models are essentially independent of the initial conditions [49]. Freezing models can also exhibit a tracker or a scaling behavior, depending whether they can provide a late-time acceleration or not, while this is not possible in scaling models. The simple exponential potential, for example, exhibits a tracking behavior and as such, it does not lead to a correct present-time behavior in which the equation of state of dark energy should be such that $\omega_{\phi} \simeq-1$ and it is then considered in general only as a suitable quintessence model with modifications of its potential [14, 50]. For the steeper potential of a generalized exponential form as introduced in Refs. 43 45, the energy density of the scalar field freezes in the past because of the Hubble damping, and later on it starts evolving and scales with the background toward the recent epoch, after which it exits to the background providing then the late-time acceleration recently. Besides having all the desirable properties as a quintessence model, it also produces a consistent early Universe inflationary cosmology in agreement with the Planck data, as also shown in Refs. [43 45.

To connect with dark matter, the authors in Ref. 44. made use of the so-called growing neutrino quintessence model [51, 52. Because of the still approximate scaling behavior of the model, by coupling the scalar field non minimally to neutrinos makes their mass to grow as the scalar field grows and this helps to trigger the late-time transition to the present accelerated expansion epoch. Here, instead, we couple the inflaton quintessential field to matter only through energy exchange effects. This, as we are going to demonstrate, when done in an appropriate way, this leads naturally to a matter-dominated regime and at the same time will make the scalar field energy density dominate again at later time, closer to the recent epoch, triggering the transition from matter domination to the current accelerated regime. This approach is very much similar to the recent studies on dark matterdark energy interactions [53-71]. This type of approach, in which interactions in the dark sector are assumed, has many appealing properties like, for example, providing a way of explaining the coincidence problem or at least alleviating it (for a thorough description of the properties of these dark sector interacting models, see, e.g., the review papers Refs. 72, 73]). Typically, the proposed forms for the interaction terms involving the dark sector are in general purely phenomenological, with many different forms treated in the literature. Here, however, we will motivate the interactions fully from the warm inflation picture. In warm inflation, different forms of dissipation coefficients have been considered (for examples, see, e.g., Refs. 74, 75]). It happens that depending on the form of the scalar potential and the dissipation coefficients, different dynamical behaviors can emerge. In the present work, we will use for the inflationary part of the dynamics the dissipation coefficient described in Refs. [27, 75, which combined with the generalized exponential form for the potential, has the property of naturally becoming negligible at the end of inflation and no further significant radiation produced from the energy of the inflaton/quintessence scalar field to light degrees of freedom being generated later on. However, we can still have interactions between the scalar field and the produced nonrelativistic matter afterward. These interactions, in fully analogy with the behavior expected from similar dissipation processes in the warm inflation dynamics, can be chosen such as to initially promote the growth of the nonrelativistic matter over radiation, creating a long enough matter-domination period after radiation domination. Then, it will subsequently contribute to making the scalar field energy density to dominate again in a mechanism similar to the one observed with other approaches with interactions in the dark sector.

The next sections of this work are organized as follows. In Sec. [II, we will present the model that we study here and its implementation in the warm inflation picture. In Sec. III we will study the early Universe inflationary dynamics of the model and give its observational predictions, contrasting them with the Planck legacy data. We will dedicate Sec. [IV] to the study of the late Universe dynamics and also obtain the different predictions of the model in this epoch. We will show that the model provides a fully consistent picture in this epoch and also contrast the results, at the background level, with those from the Planck data. Finally, in Sec. V] we will give our conclusions.

\section{MODEL}

In the warm inflation (WI) dynamics [26] the inflaton, described by a scalar field $\phi$, is coupled to radiation bath degrees of freedom, with energy density $\rho_{R}$, through a dissipation type of coefficient that is fully motivated and can be derived from microscopic physics from quantum field theory [27. The background dynamical evolution is defined by the equations

$$
\begin{aligned}
& \ddot{\phi}+3(1+Q) H \dot{\phi}+V_{, \phi}=0, \\
& \dot{\rho}_{R}+4 H \rho_{R}=3 H Q \dot{\phi}^{2},
\end{aligned}
$$

where dots denote temporal derivatives, $H$ is the Hubble parameter,

$$
H^{2} \equiv\left(\frac{\dot{a}}{a}\right)^{2}=\frac{1}{3 M_{\mathrm{Pl}}^{2}} \rho,
$$

with $\rho$ the total energy density, $a \equiv a(t)$ is the scale factor, $M_{\mathrm{Pl}} \equiv 1 / \sqrt{8 \pi G} \simeq 2.4 \times 10^{18} \mathrm{GeV}$ is the reduced 
Planck mass and $Q$ in Eqs. 2.1 and 2.2 is the dissipation ratio in $\mathrm{WI}$, defined as

$$
Q=\frac{\Upsilon(T, \phi)}{3 H},
$$

where $\Upsilon(T, \phi)$ is the dissipation coefficient in WI, which can be a function of the temperature and/or the background inflaton field, depending on the microscopic physics for WI. For instance, in Refs. [27, 42, 74-76, there are many examples of models leading to different forms for $\Upsilon(\phi, T)$. For example, in the models of Refs. [27, 74, 75, the dissipation coefficient typically scales with the temperature $T$ of the radiation bath and the background inflaton field as $\Upsilon \propto T^{3} / \phi^{2}$. In the model studied in Ref. [76] we find instead that $\Upsilon \propto T$, while in the more recent construction done in Ref. 42, it is found that $\Upsilon \propto M^{2} / T$, where $M$ is a mass scale in the model. Motivated by these functional forms found for the dissipation coefficient in WI, we can make a generic parametrization for the dissipation coefficient like

$$
\Upsilon(T, \phi)=C T^{c} \phi^{p} M^{1-c-p},
$$

where $C$ is a dimensionless constant (that carries the details of the microscopic model used to derive the dissipa- tion coefficient, e.g., the different coupling constants of the model) and numerical powers given by $c$ and $p$, which can be either positive or negative numbers (note that the dimensionality of the dissipation coefficient in Eq. (2.5) is preserved, $[\Upsilon]=[$ energy $]$ ).

Furthermore, we recall that the slow-roll parameters in WI are modified with respect to the ones in the cold inflation scenario to

$$
\begin{aligned}
\epsilon_{W I} & =\frac{\epsilon_{V}}{1+Q}, \\
\eta_{W I} & =\frac{\eta_{V}}{1+Q},
\end{aligned}
$$

where

$$
\begin{aligned}
\epsilon_{V} & =\frac{M_{\mathrm{Pl}}^{2}}{2}\left(\frac{V_{, \phi}}{V}\right)^{2}, \\
\eta_{V} & =M_{\mathrm{Pl}}^{2} \frac{V_{, \phi \phi}}{V} .
\end{aligned}
$$

Given the dissipation coefficient expressed like Eq. (2.5) and using the slow-roll approximations for the equations 2.1), 2.2 and 2.3, we can deduce, after some algebra, that $Q$ and $T / H$ have evolution equations, expressed in terms of the number of e-folds, $d N=H d t$, given, respectively, by

$$
\begin{aligned}
\frac{d \ln Q}{d N} & =\frac{2\left[(2+c) \epsilon_{V}-c \eta_{V}-2 p \kappa_{\mathrm{V}}\right]}{4-c+(4+c) Q}, \\
\frac{d \ln (T / H)}{d N} & =\frac{[7+c(Q-1)+5 Q] \epsilon_{V}-2(1+Q) \eta_{V}+(Q-1) p \kappa_{V}}{(1+Q)[4-c+(4+c) Q]},
\end{aligned}
$$

where $\kappa_{V}$ is defined as

$$
\kappa_{V}=M_{\mathrm{Pl}}^{2} \frac{V_{, \phi}}{\phi V} .
$$

The above equations determine the evolution of $Q$ and $T / H$ in the WI dynamics. Depending on the scalar field potential model and dissipation coefficient, we can have different behaviors for these quantities, for example, growing or decreasing with the number of e-folds whether the right-hand-sides of the Eqs. (2.10) and (2.11) are positive or negative, respectively. In particular, the denominator of these equations can be shown to be always positive and this is ensured when studying the dynamical stability in general of the background equations in WI [77-79]. These studies have shown that WI has a stable dynamics provided that the power $c$ in the dissipation coefficient Eq. 2.5) satisfies $-4<c<4$, which is valid in the weak $(Q \ll 1)$ and strong $(Q>1)$ dissipative regimes of WI. The sign of the numerator in Eqs. 2.10 and 2.11) can also be determined according to the model in study.

As already explained in the Introduction, in this work we will be working with the scalar potential for quintessential inflation given by the generalized exponential form 43,45

$$
V(\phi)=V_{0} \exp \left[-\alpha\left(\phi / M_{\mathrm{Pl}}\right)^{n}\right],
$$

where $V_{0}$ is the normalization of the potential and $\alpha$ is a dimensionless constant. In the conventions used in the present work, $\alpha$ is considered as a positive number and the time derivative of the scalar field is also positive, $\dot{\phi}>0$. We are also interested in potentials steeper than the simple exponential one, hence, $n>1$. When $\alpha\left(\phi / M_{\mathrm{Pl}}\right)^{n} \ll 1$, the potential is sufficiently flat to allow inflation, while as $\phi$ rolls down the potential and $\alpha\left(\phi / M_{\mathrm{Pl}}\right)^{n}$ becomes closer to 1 , the potential steepens, ending inflation. Finally, as $\phi$ continues to increase, for $\alpha\left(\phi / M_{\mathrm{Pl}}\right)^{n} \gtrsim 1$, a late-time scaling solution emerges. As discussed and explained by the authors in Ref. 44, the scalar field $\phi$ can exceed the Planck scale when $\alpha \ll 1$, yet with no related ultraviolet (UV) issues.

Since the dissipation coefficient in WI is in general a function of the temperature, it couples the scalar quintessential inflaton field directly with the radiation. Since from the observational point of view there is no reason to have this coupling present in the late Universe, 
the dissipation coefficient 2.5 must be relevant only at the early times and be negligible afterward. This is equivalent to requiring that $Q$ should be a decreasing function of time (number of e-folds) by the epoch that the scalar field enters in the scaling regime where $\alpha\left(\phi / M_{\mathrm{Pl}}\right)^{n} \gtrsim 1$. Substituting Eq. 2.13) in Eq. 2.10 for instance, we find

$$
\begin{aligned}
\frac{d \ln Q}{d N} & =-\frac{n \alpha\left(\frac{\phi}{M_{\mathrm{Pl}}}\right)^{n-2}}{4-c+(4+c) Q} \\
& \times\left[-2 c(n-1)-4 p+(c-2) n \alpha\left(\frac{\phi}{M_{\mathrm{Pl}}}\right)^{n}\right] .
\end{aligned}
$$

Thus, we find that in the scaling regime and for $c>2$, the right-hand-side of Eq. (2.14) is negative, hence, $Q$ is a decreasing function with the number of e-folds. This is in particular the case for the dissipation coefficient of Refs. 27, 74, 75, i.e., when considering $c=3, p=-2$ in Eq. (2.5). One should also note that there are other ways of making $\Upsilon$ vanish at later times, after the inflationary regime, due to the intrinsic microscopic details of the WI construction, like in Refs. [42, 76, in which after the temperature decreases to a value below a characteristic scale of the model, the dissipative effects can shut down. However, this is mostly dependent of the microscopic physics involved and in the present work, we adopt a more model independent approach, in which the coupling between the scalar field and radiation can naturally become negligible (and effectively inefficient) after inflation as a result of the background dynamics. Hence, from now on we will assume that the early Universe inflationary dynamics is dominated by the dissipation coefficient with the cubic dependence on the temperature,

$$
\Upsilon_{\text {cubic }}=C_{\text {cubic }} \frac{T^{3}}{\phi^{2}},
$$

and the dissipation ratio that couples the scalar quintessential inflaton field to radiation is then $Q \equiv$ $\Upsilon_{\text {cubic }} /(3 H)$.

In addition to Eqs. (2.1) and 2.2 for the background dynamics of $\phi$ and $\rho_{R}$, we should also complement them with the evolution equation for the matter energy density, $\rho_{m}$, so to be able to properly describe the late-time evolution of the Universe. The matter energy density can also be split into the dark matter and baryon energy densities, $\rho_{D M}$ and $\rho_{b}$, respectively, such that $\rho_{m} \equiv \rho_{D M}+\rho_{b}$. In the present work we will not make the distinction between the background evolution for each of these components separately and we will treat only the evolution of the total matter energy density, i.e., we will treat the dark matter and baryons as a single matter fluid. One should also recall that baryons total about one-sixth of the present total matter distribution, thus, we expected that by not making a distinction between dark matter and baryons, we should not be incurring in any significant error in the numerical evaluations in the next sections. This should, in particular, be true at least at the background level, which is the case we focus on this work.
As far as dark matter production is concerned, WI itself can have the mechanisms needed for generating it. This can happen either through the intrinsic dissipative dynamics inherent of the WI picture, or also as a consequence of it, like, e.g., the inflaton itself being a stable remnant behaving at later times as dark matter 80 , 81. For some decaying vacuum energy models that can possibly behave like in WI, see, e.g., Ref. [82]. The possibility of matter generation (through a baryogenesis scenario) in WI was explored before in Refs. 83, 84. In particular, iRef. 85] studied in detail the observational implications of this scenario, with the prediction that the matter production in WI would lead to fully anticorrelated isocurvature perturbations. These previous studies motivate us to treat the matter evolution dynamics as concomitant with those from the scalar field and the radiation fluid. Indeed, the dissipation model studied in Refs. $[83,85$, was actually the same that leads to the dissipation coefficient of the form of Eq. 2.15. The recent models of dark matter and dark energy interactions [72, 73] also motivate us to consider similar energy exchange terms involving the dark sector. Here, however, we motivate these terms from the WI picture. Indeed, we can show that by simply replacing the radiation energy density by the matter density, $T \propto \rho_{R}^{1 / 4} \rightarrow \rho_{m}^{1 / 4}$, we can construct dissipation ratios $Q_{\rho_{m}}$ with dynamical behavior similar to the ones seen in Eq. 2.14). We will use this generic property in our construction below. Motivated by the above remarks, here we propose the complete set of background equations involving the quintessential scalar field, the radiation fluid and the matter energy densities as given, respectively, by

$$
\begin{aligned}
& \ddot{\phi}+3(1+Q) H \dot{\phi}+\Upsilon_{\rho_{m}} \dot{\phi}+V_{, \phi}=0, \\
& \dot{\rho}_{R}+4 H \rho_{R}=3 H Q \dot{\phi}^{2}, \\
& \dot{\rho}_{m}+3 H \rho_{m}=\Upsilon_{\rho_{m}} \dot{\phi}^{2},
\end{aligned}
$$

where $\Upsilon_{\rho_{m}}$ describes the energy exchange term between the quintessential scalar field and matter energy density and we parametrize it in the form

$$
\Upsilon_{\rho_{m}}=c_{m} \frac{\rho_{m}^{3 / 4}}{\phi^{2}}+\frac{M^{2}}{\rho_{m}^{1 / 4}},
$$

where $c_{m}$ is a dimensionless constant and $M$ is another constant with mass dimension. The form of the first term in Eq. 2.19) is closely motivated by the Eq. 2.15). Similarly to the behavior found for the dissipation coefficient of the form Eq. 2.15), the first term in Eq. 2.19) displays a growing dynamics during inflation, setting an abundance for the matter density until around the beginning of the radiation-domination regime, after which it decays with time. On the other hand, the second term in Eq. 2.19 displays opposite behavior, decreasing throughout inflation but later on growing with time. Given appropriate parameters $c_{m}$ and $M$, we can arrange a similar behavior found, e.g., in the case of nonminimal couplings of the scalar field to matter [53. With the second term in Eq. 2.19), as it grows at later times, 
it will eventually provide an extra friction force on the quintessence scalar field and help making $\phi$ acquire a negative equation of state, signaling the beginning of the dark energy (quintessence) domination epoch. As far the dependences on $\rho_{m}$ in Eq. 2.19) are concerned, we could also think in a term similar to a dissipation coefficient that is proportional to the temperature in the WI scenario [76]. Then, by the same analogy as assumed in the construction of the terms in Eq. (2.19), by taking $T \rightarrow \rho_{m}^{1 / 4}$, such a term would have a behavior similar to the second term on the right-hand side of Eq. (2.19), growing at the end of the inflationary regime and leading the energy density of the scalar field to dominate at present. However, the growth is much slower than provided by the second term in Eq. 2.19), making the transition from matter domination to the acceleration regime happen at a much higher redshift than is observationally acceptable (likewise, the matter domination regime is too short). Such a term in Eq. (2.19) is then rendered unacceptable. Likewise, we could look at the first term in Eq. 2.19) with a larger power in the matter energy density, but then we face the opposite behavior, a potential larger matter domination regime and much later transition to the acceleration regime (and also possibly instabilities issues as far as a dynamical system analysis is concerned, similar to what is seen in WI with dissipation coefficients with a large power in the temperature 77 79]). In this sense, the dependences in Eq. (2.19) seem a middle term between these extreme behaviors.

In the next sections, we will show explicitly how each of the dissipative terms appearing in Eqs. 2.16), 2.17) and (2.18), and given by Eqs. (2.15) and (2.19), help produce a consistent picture for the evolution of the scalar (quintessential inflaton) field and the radiation and matter constituents, from the primordial inflation time up to the recent epoch. We will start by first studying the inflationary early Universe epoch.

\section{EARLY UNIVERSE INFLATIONARY COSMOLOGICAL DYNAMICS}

The potential (2.13) can support an inflationary regime in its flat region, $\alpha\left(\phi / M_{\mathrm{Pl}}\right)^{n} \ll 1$. The predictions for this model can be contrasted with those from the recent CMB observations, e.g., from the Planck data [86. We are, in particular, interested in the tensor-to-scalar ratio $r$ and the spectral tilt $n_{s}$, defined, respectively, by

$$
r=\frac{\Delta_{T}}{\Delta_{\mathcal{R}}}
$$

and

$$
n_{s}-1=\lim _{k \rightarrow k_{*}} \frac{d \ln \Delta_{\mathcal{R}}\left(k / k_{*}\right)}{d \ln \left(k / k_{*}\right)},
$$

where $\Delta_{\mathcal{R}}$ is the primordial scalar curvature power spectrum and $\Delta_{T}$ is the tensor power spectrum. Quantities with a subindex $*$ mean that they are evaluated at the Hubble radius crossing, $k_{*}=a_{*} H_{*}$. The Planck Collaboration 86] gives for $r$ the upper bound, $r<0.056$ (95\% CL, Planck TT,TE,EE+lowE+lensing+BK15, at the pivot scale $k_{p}=0.002 / \mathrm{Mpc}$ ), while for the spectral tilt the result is $n_{s}=0.9658 \pm 0.0040(95 \% \mathrm{CL}$, Planck TT, TE,EE+lowE+lensing + BK15+BAO+running).

Furthermore, the normalization of the primordial scalar curvature power spectrum, at the pivot scale $k_{*}$, is given by $\ln \left(10^{10} \Delta_{\mathcal{R}}\right) \simeq 3.047$ (TT,TE,EElowE+lensing+BAO $68 \%$ limits), according to the Planck Collaboration [1] and this is the value we will assume in all our numerical simulations, in particular for finding the normalization $V_{0}$ of the potential Eq. 2.13).

In the cold inflation scenario, i.e., in the absence of dissipative effects and no radiation bath during inflation, $\Delta_{\mathcal{R}}$ and $\Delta_{T}$ are given, respectively, by the standard expressions [87]

$$
\begin{aligned}
& \Delta_{\mathcal{R}}=\left(\frac{H^{2}}{2 \pi \dot{\phi}}\right)^{2}, \\
& \Delta_{T}=\frac{2 H^{2}}{\pi^{2} M_{\mathrm{Pl}}^{2}} .
\end{aligned}
$$

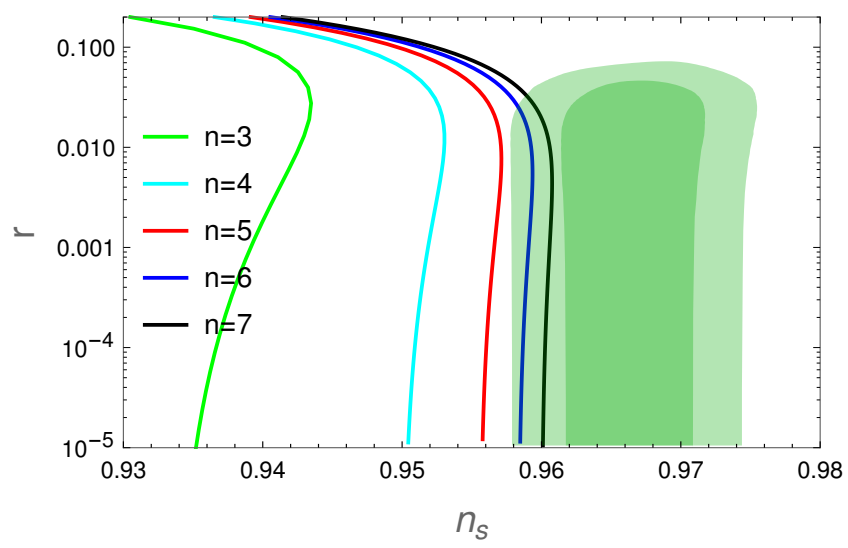

FIG. 1. The spectral index $n_{s}$ and the tensor-to-scalar ratio $r$ in the plane $\left(n_{s}, r\right)$, in the cold inflation scenario (i.e., in the absence of dissipative effects) for different values of the exponent $n$ and constant $\alpha$, such that $10^{-12} \leq \alpha \leq 10^{-1}$ ( $\alpha$ increases when going from small to larger values of $r$ ). The shaded areas are for the $68 \%$ and $95 \%$ C.L. results from Planck 2018 $(\mathrm{TT}+\mathrm{TE}+\mathrm{EE}+$ lowE+ lensing $+\mathrm{BK} 15+\mathrm{BAO}$ data $)$.

In the Ref. 44, the authors have studied in detail the predictions for the model with the potential given by Eq. 2.13), including approximate analytical expressions for $r$ and $n_{s}$ in the cold inflation case. For completeness, in Fig. 1 we show these results for different values of the power $n$ in Eq. 2.13). The dimensionless constant $\alpha$ in the potential varies, $10^{-12} \leq \alpha \leq 10^{-1}$. The values of $\alpha$ increase when going from small to larger values of $r$, which then determines the curves in Fig. 1. For this 
figure, we have considered $N_{*}=60$ for the number of e-folds before the end of inflation.

One notices from Fig. 1 that only for $n>5$ can one have results for the spectral tilt compatible with the recent observational data at the $2 \sigma$ level. This situation can change considerably in the WI case. Because of dissipation and the presence of a radiation bath, the primordial scalar power spectrum given by Eq. (3.3) changes, while the tensor spectrum Eq. (3.4) remains unchanged. The primordial power spectrum for WI at horizon crossing can be expressed in the form (see, e.g., Refs. [88 90])

$$
\Delta_{\mathcal{R}}\left(k / k_{*}\right)=\left(\frac{H_{*}^{2}}{2 \pi \dot{\phi}_{*}}\right)^{2} \mathcal{F}\left(k / k_{*}\right)
$$

where the function $\mathcal{F}\left(k / k_{*}\right)$ in Eq. 3.5 is given by

$$
\mathcal{F}\left(k / k_{*}\right) \equiv\left(1+2 n_{*}+\frac{2 \sqrt{3} \pi Q_{*}}{\sqrt{3+4 \pi Q_{*}}} \frac{T_{*}}{H_{*}}\right) G\left(Q_{*}\right),
$$

where $n_{*}$ denotes the inflaton statistical distribution due to the presence of the radiation bath and $G\left(Q_{*}\right)$ accounts for the effect of the coupling of the inflaton and radiation fluctuations 91 93. $G\left(Q_{*}\right)$, in general, can only be determined by numerically solving the set of perturbation equations in WI. However, in the weak dissipative regime of WI, where $Q_{*} \ll 1$, these coupling effects on the scalar curvature power spectrum are negligible and we can safely consider $G\left(Q_{*}\right) \simeq 1$ in Eq. (3.6). In the present work we will restrict to this regime, which will suffice for us to obtain our main results. For a note on this point of considering the weak dissipative regime, one should, however, note that evading the recent so-called Swampland conjectures, one typically requires going in the strong dissipative regime of WI, $Q \gg 1$, as shown in Refs. [40, 42]. With the generalized exponential potential Eq. 2.13 we can find parameter regimes supporting $Q \gg 1$, but in the present work we will not explore this region of parameters but leave this open for future studies. In fact, even the simple exponential potential (with $n=1$ ) is also shown to support the strong dissipative regime and also lead to consistent inflationary observables, provided that we extend the system beyond the general relativity case, e.g., in the context of extra dimensions and the braneworld extension, as shown recently in Ref. [94, in which all the swampland conjectures can be overcome.

Before presenting our results for the WI case, let us comment on the choice of the initial conditions in our problem. Deep in the inflationary regime, we can use the slow-roll equations derived from Eqs. (2.16) and (2.17). The matter energy density is negligible during that time and can be neglected (including $\Upsilon_{\rho_{m}}$ ). With these equations and also using the expression for the scalar curvature power spectrum Eq. (3.5) and the corresponding CMB normalization, we can fix the normalization $V_{0}$ in the inflaton potential and find the appropriate initial conditions leading to the required $N_{*}$ e-folds of inflation be- fore its end, which is the point in the inflationary evolution relevant for obtaining $r$ and $n_{s}$. In particular, the value for $\phi_{*}$ obtained is always a bit farther away to the right from the inflection point of the potential (when $n$ is odd), or away (also to the right) from the top of the potential hill (when $n$ is even), since otherwise we could be starting with initial conditions leading to a much larger value of e-folds of inflation and having to wait some long time until the relevant instant $N_{*}$ when the scales of interest leave the Hubble radius. Here, as also in many other works on inflation, including Ref. 44, which made explicit studies of the present generalized exponential inflaton potential, we do not address how exactly the system achieves the conditions necessary for inflation. Physically viable suggestions for mechanisms setting the appropriate conditions for inflation are, for example, through some earlier phase transition, or through thermal and dissipative effects acting in the preinflationary phase, as suggested and developed in Ref. [95.

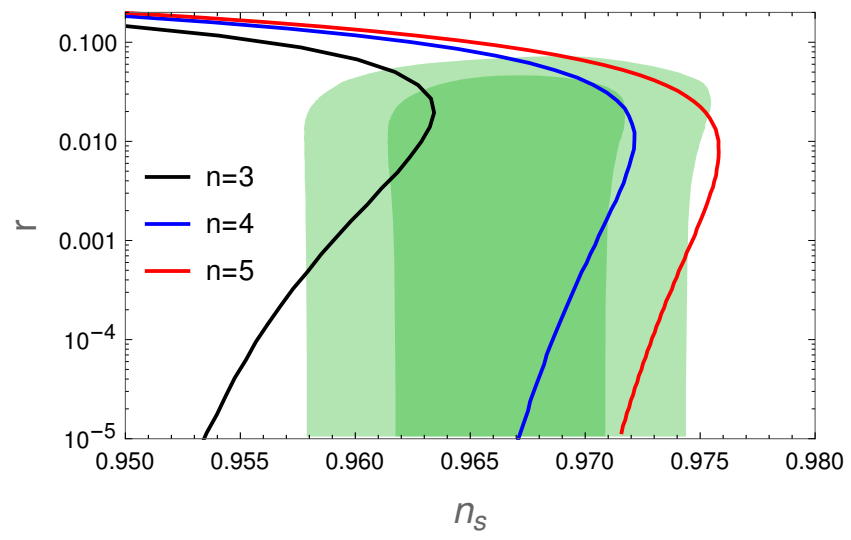

FIG. 2. Similar to Fig. 1, but in the WI scenario, for a dissipation ratio $Q_{*}=5 \times 10^{-7}$. Only the $n=3,4,5$ cases are shown.

By performing the analysis in the WI case, with scalar power spectrum given by Eq. (3.5) and setting $G\left(Q_{*}\right)=1$ in Eq. (3.6), which is justified for $Q_{*} \ll 1$, we show in Fig. 2 the results equivalent to Fig. 1. In Fig. 2 we have fixed the dissipation ratio at the value $Q_{*}=5 \times 10^{-7}$. Though very small, it is already large enough to deform the curves (with varying $\alpha$ ) shown in the previous Fig. 1. In particular, the models with $n=3$ and $n=4$, which were excluded beforein the cold inflation scenario, are now found to be consistent with the Planck data for a range of $\alpha$ values ${ }^{2}$.

We also recall from Eq. (2.14) that $Q$ is evolving with the number of e-folds. Thus, even though we might have

\footnotetext{
${ }^{2}$ However, we find no values for the dissipation and coefficient $\alpha$ such to get the case with $n=2$ inside the Planck confidence regions, which has a $n_{s}$ that is always too red tilted.
} 
a very small $Q_{*}$ at the instant $N_{*}$ e-folds before the end of inflation (and still also be inside the WI regime, where $T>H$ ), by the end of inflation, and before the scaling regime for which $\alpha\left(\phi / M_{\mathrm{Pl}}\right)^{n} \gtrsim 1, Q_{\text {end }}$ can be much larger than $Q_{*}$. This is in fact what facilitates the transition from the inflationary regime to the radiationdominated one. We illustrate this behavior in Fig. 3, taking as an illustration, the case with $n=3$ in Eq. (2.13) and for a dissipation ratio $Q_{*}=6.24 \times 10^{-6}$, which is one of the cases explicitly studied in the next section (see Table II.

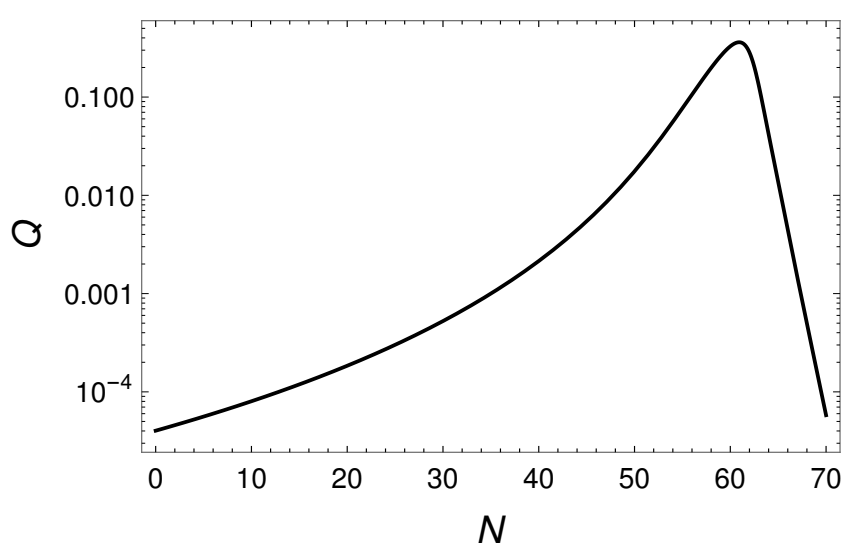

FIG. 3. The evolution of the dissipation ratio $Q$ with the number of e-folds for the model $n=3$ and parameters given in Table I

In the model and parameters considered in Fig. 3, we have $N_{*} \simeq 61.6$. We see that during inflation $Q$ grows by more than 4 orders of magnitude and at the end of inflation, $Q_{\text {end }} \simeq 0.33$. Soon after that and as anticipated in the discussion following Eq. (2.14), with the scalar field satisfying $\alpha\left(\phi / M_{\mathrm{Pl}}\right)^{n} \gtrsim 1$, the value of $Q$ quickly drops down. It is also useful to show the dynamics for the inflaton kinetic $K=\dot{\phi}^{2} / 2$, the potential $V(\phi)$, and the radiation $\rho_{R}$ energy densities. This is shown in Fig. 4

We notice from the results shown in Fig. 4 that before the system transits to the radiation-dominated phase, there is a small regime in which the kinetic energy of the scalar field is the largest energy component. This kinetic regime lasts however a very short time, however, about $\sim 0.2$ e-folds in the present example. This is a characteristics of the WI dynamics and also seen in the warm quintessential inflation studied e.g. in Ref. [29].

Having established that one can have a consistent inflationary regime in the WI dynamics with the primordial steep potentials of the form of Eq. (2.13), let us now analyze the late-time dynamics for the model, with background equations given by Eqs. 2.16, 2.17) and (2.18, with dissipation coefficients given by Eqs. 2.15)

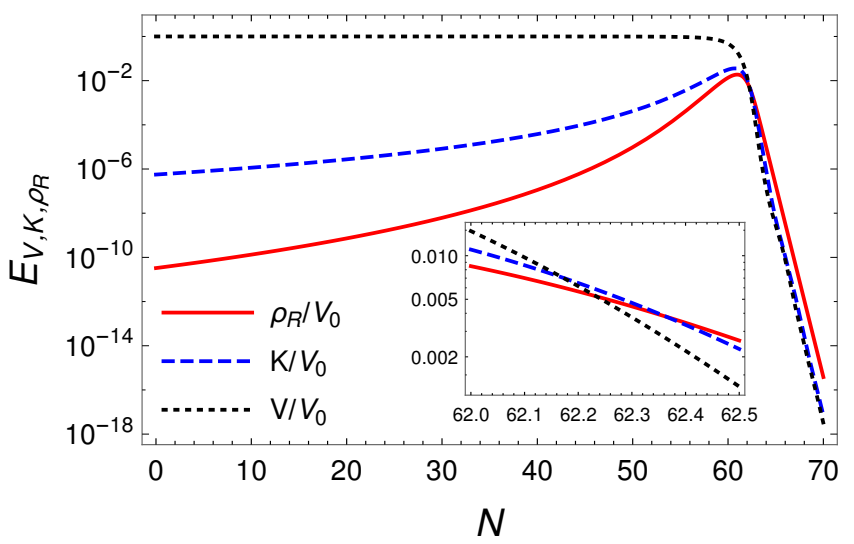

FIG. 4. The evolution of the kinetic $K=\dot{\phi}^{2} / 2$, of the potential $V(\phi)$, and for the radiation $\rho_{R}$ energy densities, for the same model and parameters used in Fig. 3 . All energy densities are normalized by $V_{0}$, where $V_{0} \simeq 1.62 \times 10^{15} \mathrm{GeV}$ for the present model parameters. The inset zooms in a region right after inflation.

and 2.19. We will focus on three models in particular, in which $n=3,4,5$.

\section{LATE-TIME UNIVERSE DYNAMICS}

For the late-time dynamics, we will work with three explicit examples, considering the cases of $n=3,4,5$ in the potential Eq. 2.13. The constant $\alpha$ in the potential and the dissipation ratio $Q_{*}$ determining the inflationary dynamics, such as the value for spectral tilt $n_{s}$ that we find is always close to the central value from the Planck data, i.e., $n_{s} \simeq 0.965$, are chosen. The number of e-folds of inflation $N_{*}$ between the moment the relevant scales with wave number $k_{*}$ leave the Hubble radius and reenter around today, is defined by the relation 96 .

$$
\frac{k_{*}}{a_{0} H_{0}}=e^{-N_{*}} \frac{T_{0}}{T_{\text {end }}} \frac{H_{*}}{H_{0}},
$$

where $a_{0}$ and $H_{0}$ are the today values for the scale factor and Hubble parameter, respectively. $H_{*}$ is the Hubble parameter during inflation and $T_{\text {end }}$ and $T_{0}$ are the temperature at the beginning of the radiation-dominated regime and the CMB temperature today, respectively. Typically, in the cold inflation scenario $T_{\text {end }}$ is difficult in general to obtain given that it depends of the details of the reheating process after inflation. In WI, however, this is much simplified, since the transition from the end of inflation to the radiation-dominated regime is smooth and $T_{\text {end }}$ is simply the temperature at the end of WI. Therefore, we can self-consistently obtain $N_{*}$ from Eq. (4.1).

In this work, we take the convention that $a_{0}=1$ (likewise, the redshift parameter today is $\left.z_{0}=0\right)$. For the Hubble parameter today, we will assume the Planck result, $H_{0}=67.66 \mathrm{~km} \mathrm{~s}^{-1} \mathrm{Mpc}^{-1}$ [from the Planck Collaboration [1], TT,TE,EE-lowE+lensing+BAO $68 \%$ lim- 
its, $\left.H_{0}=(67.66 \pm 0.42) \mathrm{km} \mathrm{s}^{-1} \mathrm{Mpc}^{-1}\right]$. Likewise, for the CMB temperature today we assume the value $T_{0}=$ $2.725 \mathrm{~K}=2.349 \times 10^{-13} \mathrm{GeV}$. The value of $T_{0}$ will fix the total number of e-folds of expansion, $N_{\text {total }}$ from the instant $N_{*}$ deep in the inflationary regime until today. We still need to find the constants $c_{m}$ and $M$ in Eq. 2.19. These can be unambiguously found by requiring that we obtain the appropriate values for the dark matter and dark energy density ratios today, $\Omega_{m, 0}$ and $\Omega_{\mathrm{DE}, 0}$, respectively. For these values, we use again the dataset TT,TE,EE-lowE+lensing+BAO $68 \%$ limits from the Planck Collaboration, which give the values $\Omega_{m, 0}=0.3111 \pm 0.0056$ and $\Omega_{\mathrm{DE}, 0}=0.6889 \pm 0.0056$ (see, e.g., Table 2 of Ref. [1]). Our model parameters are determined such as to lead to values for $\Omega_{m, 0}$ and $\Omega_{\phi, 0}$ that are between the range found in these observational results, which are here taken to be our fiducial values. Likewise, the fractional density in radiation, $\Omega_{r}$, is always at a level consistent with its value today, $\Omega_{r, 0} \sim 8 \times 10^{-5}$ (including neutrinos).

TABLE I. The three model examples considered, along with the respective parameters and the relevant cosmological quantities (see text) obtained from them.

\begin{tabular}{|c|c|c|c|c|c|c|c|c|}
\hline Model & $n_{s}$ & $r$ & $N_{*}$ & $N_{\text {total }}$ & $c_{m}$ & $M(\mathrm{eV})$ & $z_{\mathrm{DM}-\mathrm{DE}}$ & $\Omega_{\phi}\left(z_{\mathrm{rec}} \approx 1100\right)$ \\
\hline $\begin{array}{c}n=3 \\
\alpha=0.05 \\
Q_{*}=4.03 \times 10^{-5}\end{array}$ & 0.9652 & $6.24 \times 10^{-6}$ & 61.6 & 125.2 & 0.0668 & $1.48 \times 10^{-16}$ & 0.6135 & 0.00186 \\
\hline $\begin{array}{c}n=4 \\
\alpha=0.01 \\
Q_{*}=2.55 \times 10^{-7}\end{array}$ & 0.9659 & $9.24 \times 10^{-5}$ & 62.1 & 126.6 & 0.0455 & $2.72 \times 10^{-16}$ & 0.6051 & 0.00056 \\
\hline $\begin{array}{c}n=5 \\
\alpha=4 \times 10^{-4} \\
Q_{*}=8.73 \times 10^{-8}\end{array}$ & 0.9657 & $4.24 \times 10^{-4}$ & 62.6 & 127.3 & 0.0723 & $2.99 \times 10^{-16}$ & 0.6050 & 0.00047 \\
\hline
\end{tabular}

Our results for the three examples using $n=3,4,5$ are summarized in Table II. We also quote the results obtained for the redshift at the transition from dark matter to dark energy domination, $z_{\mathrm{DM}-\mathrm{DE}}$. In Table II we also give the value for the density parameter for the quintessential scalar field close to recombination, $\Omega_{\phi}\left(z_{\text {rec }} \approx 1100\right)$, which is a useful result to be compared with early dark energy constraints.

The values obtained for $M$ and given in Table I] interestingly, they fall in the ballpark for the masses of light axionlike pressureless cold dark matter particles [97. In principle we could also directly write $M$ in terms of some typical mass scale for dark matter, up to some constant $c_{M}$, e.g., $m_{\mathrm{DM}}=c_{M} M$. The two dissipation terms in Eq. 2.19 in principle should both come from similar physical processes, thus, the constants $c_{m}$ and $c_{M}$ are not expected to differ in value by too disparate numbers. Even when setting, for example, $c_{M} \ll c_{m}, m_{\mathrm{DM}}$ can reach the mass range of the so-called fuzzy cold dark matter 98, $10^{-33} \mathrm{eV} \lesssim m_{\mathrm{DM}} \lesssim 10^{-18} \mathrm{eV}$. Even though we did not make any assumptions here about the underline dark matter being produced (except that which is cold), the values of $M$ obtained and shown in Table I are quite suggestive. Despite that the mass scale $M$ might have some physically well-motivated value, its value is, in principle, not related to any other natural energy scale in our model. Thus, its smallness can still be associated with some level of fine-tuning due to $M$ being much smaller than the vacuum energy density in the $\Lambda$ CDM model, $\rho_{\Lambda}^{1 / 4} \sim 10^{-3} \mathrm{eV}$, but still much larger than $H_{0} \sim 10^{-33} \mathrm{eV}$.

Early dark energy can potentially change the expansion history in the early and also in the late Universe. It can affect galaxy formation, big bang nucleosynthesis and the CMB anisotropies. The strongest bound to date come from the CMB data [99], which sets the upper bound $\Omega_{\phi}\left(z_{\text {rec }}\right) \lesssim 0.0036$ (at $95 \%$ confidence level, for Planck TT,TE,EE-lowP+BSH) on the amount of dark energy (here described by quintessence) at the time of recombination, $z_{\text {rec }} \approx 1100$. Our results for $\Omega_{\phi}\left(z_{\text {rec }}\right)$ for

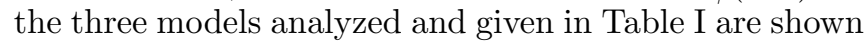
to satisfy this upper bound.

The redshift at the transition from dark matter to dark energy domination, $z_{\mathrm{DM}-\mathrm{DE}}$, has been considered as a possible additional cosmological parameter. Though it is very difficult to precisely constrain $z_{\mathrm{DM}-\mathrm{DE}}$ (see, e.g., Ref. [100] for a recent analysis), it is generically assumed that any model describing dark energy and the current cosmological data, should have a value such that $0.5<z_{\mathrm{DM}-\mathrm{DE}}<1$. Again, our results for the three models analyzed produce acceptable values for $z_{\mathrm{DM}-\mathrm{DE}}$, as shown in Table 1 .

In Fig. 5, we show the complete evolution for each of the energy density fractions, $\Omega_{\phi}, \Omega_{m}$ and $\Omega_{r}$, for the 


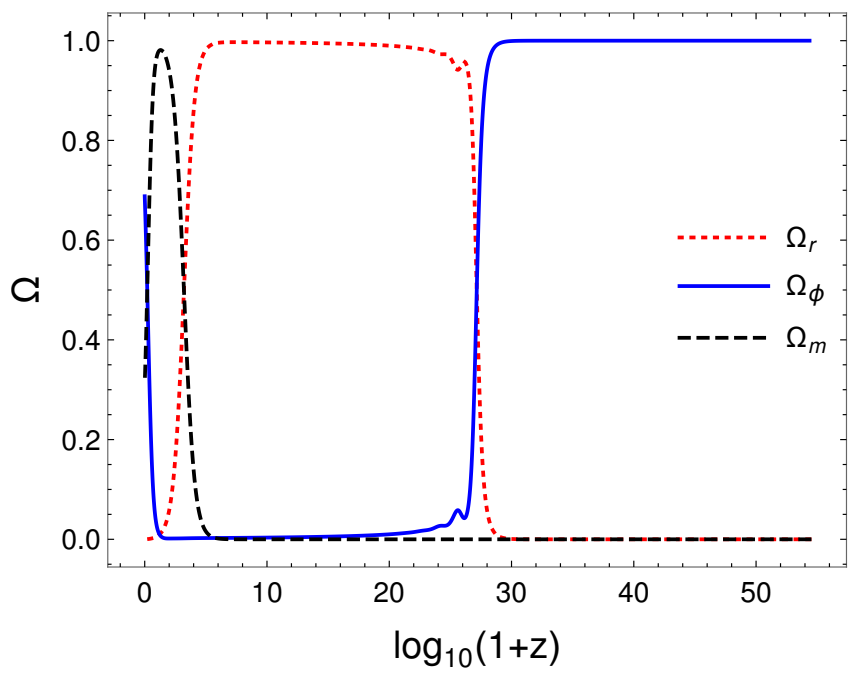

FIG. 5. The energy density fractions, $\Omega_{\phi}, \Omega_{m}$ and $\Omega_{r}$, as a function of the redshift, for the model $n=3$ and parameters given in Table I

quintessence scalar field, the matter and radiation fluid components. This is shown for the model with $n=3$ with the parameters given in Table I. The other two models have very similar results and, hence, we do not show them here.

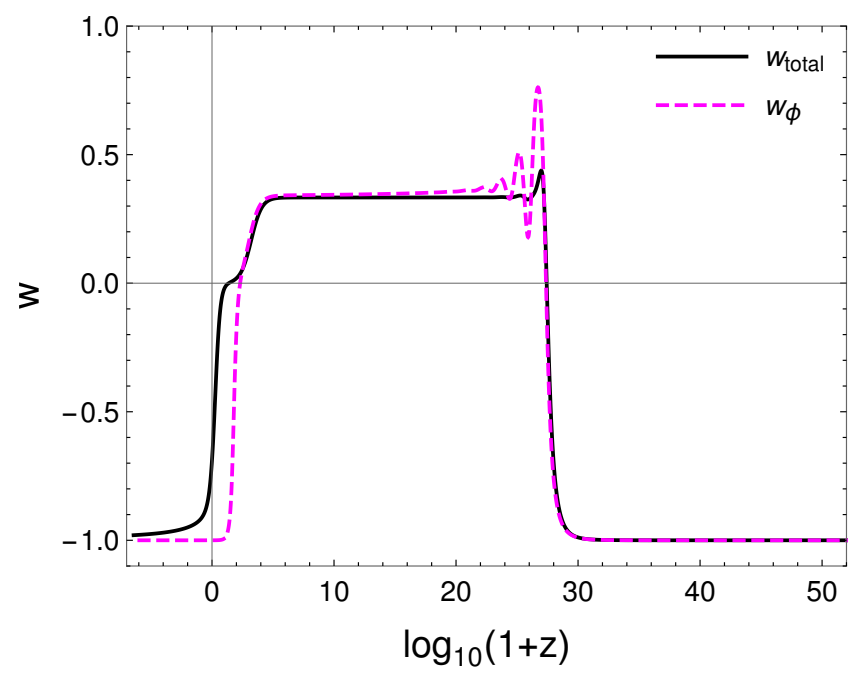

FIG. 6. The equations of state for the quintessence scalar field, $w_{\phi}$, and for the whole Universe, $w_{\text {total }}$, as a function of the redshift, for the model $n=3$ and parameters given in Tab. I]

In Fig. 6, we show the equation of state (EOS) for the quintessence scalar field, $w_{\phi}$, and for the whole Universe, $w_{\text {total }}$. Again, we show the results only for the model with $n=3$, with the other two models having very similar results. We note from the results shown in Fig. 6 that the Universe goes from the inflationary regime to the radiation-dominated one with a very short intermediate kination period, in which $1 / 3<w_{\text {total }} \leq 1$. This is quite different from typical inflation quintessential models, in which one usually finds a rather long kination period and the Universe expands like stiff matter, $\rho \propto 1 / a^{6}$. Here, however, because of the different dissipative effects, first from the WI intrinsic dissipation, Eq. 2.15), and the subsequent quintessence-dark matter interaction, Eq. 2.19), their effects are fundamental to first leading to a smooth transition from the inflationary regime to the radiation-dominated one and then to maintain the Universe in a radiation $\operatorname{EOS} w_{\text {total }}=1 / 3$, subsequently, except for a very short period after inflation 3 , as shown in Fig. 6, with $w_{\text {total }}$ never reaching 1 , as expected for a fully developed kination period. This is quite an important result, since large kination periods can be potentially dangerous in many quintessence models. In a model like that given by Eq. 2.13), in the absence of particle production mechanisms like the one of WI, one has to rely on gravitational particle production. The produced gravitational radiation can then be boosted by the kination period and it can potentially dominate by the time of big bang nucleosynthesis and can even challenge a successful nucleosynthesis [45, 101]. It is quite satisfying to see that in the present model we do not have any such issues. In fact, we do not have to rely at all on gravitational particle production. Particle production in the present case is all from quasiadiabatic motion of the scalar field $\phi$ along its potential. This mechanism of particle production, typical of the WI scenario (contrary to particle production from the quantum vacuum) is assured by the presence of the thermal bath, which was first discussed in connection to inflation by Hosoya and Sakagama in Ref. [102] and also by Morikawa and Sasaki in Ref. [103. A proper quantum field theory interpretation of this particle production mechanism in a thermal bath was later given by Moss and Graham in Ref. [104, in particular leading to a dissipation coefficient of the form of Eq. 2.15). For an additional remark concerning the results for the EOS for the quintessence scalar field, $w_{\phi}$, and shown in Fig. 66 let us note that a time-varying EOS for dark energy, of the form $w(a)=w_{0}+(1-a) w_{a}$, has been assumed by several works (see, e.g., Ref. [1]). Given that the quintessence field in our model follows a quick freezing solution after radiation domination and still in the matter dominated regime, as can be noticed

\footnotetext{
3 The oscillating behavior in the EOS for the scalar field at the end of the inflationary phase and commencement of the radiation one (which also manifests with less intensity in $w_{\text {total }}$ ), is a reminiscence of the kination tendency typical of the quintessential inflation models of the type studied here (this very small kination regime is also seen in Fig. 4 and discussed in there). These features become less prominent for larger values of $n$ and they also tend to be damped away the larger is the dissipation ratio $Q_{*}$, which leads to a much smoother transition between the end of inflation to the radiation-dominated regime.
} 
from Fig. 6, we have that $w_{0} \simeq-1$ and $w_{a} \simeq 0$ and, then, the scalar field $\phi$ late-time behavior, by the present epoch $\left(a_{0}=1\right)$, is practically indistinguishable from that of a cosmological constant.

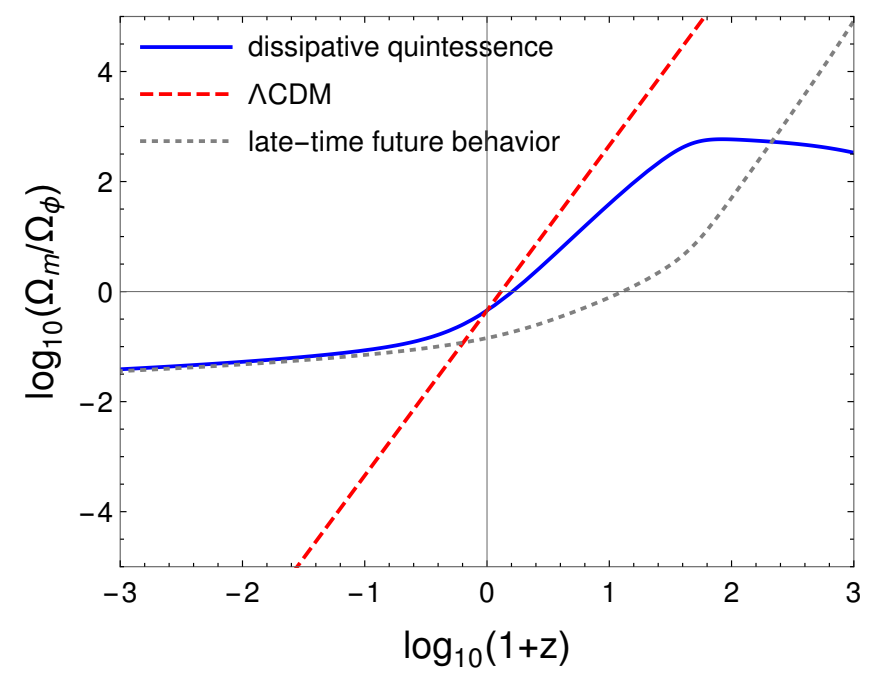

FIG. 7. The ratio of dark matter and quintessence energies densities, $\rho_{m} / \rho_{\phi} \equiv \Omega_{m} / \Omega_{\phi}$, as a function of the redshift, for the model $n=3$ and parameters given in Table I (solid line) and the analogous quantity for the $\Lambda \mathrm{CDM}$ model (dashed line). The dotted line indicates the late-time result given by Eq. 4.5.

Finally, in Fig. 7, we show the ratio of dark matter and quintessence energies densities, $\rho_{m} / \rho_{\phi} \equiv \Omega_{m} / \Omega_{\phi}$, as a function of the redshift and compare the result with the one from the $\Lambda \mathrm{CDM}$ model. We notice that the slope of the curve from the quintessence model $n=3$ (again the other two cases shown in Table I give similar results) is smaller than the one from the $\Lambda \mathrm{CDM}$ model. In particular, $d\left(\rho_{m} / \rho_{\phi}\right) /\left.d t\right|_{z_{0}=0}<H_{0}$, which has been seen also in dark energy-dark matter interaction models [58, 73]. The variation $d\left(\rho_{m} / \rho_{\phi}\right) / d t$ in the present model being slower than that of the $\Lambda \mathrm{CDM}$ model can alleviate the coincidence problem seen in the latter [58, 73. We also note from Fig. 7 that the scalar field does not quite follow a scaling dynamics with the matter density in the matter dominated regime, which is consistent with what is seen also in Fig. 6. After matter-radiation equality the scalar field loses the scaling with the dominant energy density. However, as we move toward the very distant future, $z<0$, a late-time behavior relating $\rho_{m}$ with $\rho_{\phi}$ emerges. As we extend the dynamics toward the future, $z<0$, a slow-roll approximation for the background dynamics for $\phi$ and $\rho_{m}$ can be used. In this case, as the dissipation ratio $Q$ and the radiation energy density $\rho_{R}$ are completely negligible, we can approximate the Eqs. 2.16 and 2.18, respectively, as

$$
\begin{aligned}
& \dot{\phi} \approx-\frac{V_{, \phi}}{\Upsilon_{\rho_{m}}} \approx-\frac{V_{, \phi}}{M^{2}} \rho_{m}^{1 / 4}, \\
& \rho_{m} \approx \frac{\Upsilon_{\rho_{m}}}{3 H} \dot{\phi}^{2} \approx \frac{\left(V_{, \phi}\right)^{2}}{3 H M^{2}} \rho_{m}^{1 / 4},
\end{aligned}
$$

where we have used in the above equations that at late times the dominant contribution in $\Upsilon_{\rho_{m}}$ is the last term in Eq. 2.19). Thus, using that at late times $z<0$, $H^{2} \sim \rho_{\phi} /\left(3 M_{\mathrm{Pl}}^{2}\right)$ and $\rho_{\phi} \sim V(\phi)$, hence, from Eq. 4.3 , we have that

$$
\rho_{m}^{3 / 2} \approx \frac{\left(V_{, \phi}\right)^{4} M_{\mathrm{Pl}}^{2}}{3 M^{4} V(\phi)},
$$

and, using also the expression for the potential Eq. 2.13, we can finally obtain that

$$
\frac{\rho_{m}}{\rho_{\phi}} \approx\left(\frac{\alpha n \phi^{n-1}}{M_{\mathrm{Pl}}^{n-1}}\right)^{8 / 3} \frac{V(\phi)}{\left(3 M^{4} M_{\mathrm{Pl}}^{2}\right)^{2 / 3}} .
$$

From Fig. 7 we see that the result given by Eq. 4.5, indicated by the dotted line, agrees quite well with the full numerical result when $\log _{10}(1+z)<-1$.

\section{CONCLUSIONS}

In this work we have proposed a dissipative quintessential inflation model that is able to fully describe both the early inflationary Universe and the present epoch. The early Universe dynamics is realized in the warm inflation scenario. The late dynamics is motivated by the recent dark energy-dark matter interaction models, however, differently from what is adopted in those models, here we motivate the interaction term by the dissipative terms typically found in warm inflation. By doing so, we have defined a new type of interacting model connecting both early- and late-time dynamics. Both the inflationary and the late-time periods have been described in terms of a steep class of generalized exponential potentials. The role of the different forms of dissipation terms adopted have been shown to be fundamental to lead to a consistent picture.

We have obtained values for the tensor-to-scalar ratio and the spectral tilt of the primordial spectrum that are consistent with the ones obtained by the Planck legacy data. Likewise, we have an appropriate description of the late-time dynamics that avoids any strong fine-tuning of parameters, as typically plagues many quintessence models in general. Our results share many similarities with other dark sector types of interaction models, including an alleviation of the coincidence problem.

Our model also displays some very welcome features. For instance, the WI dynamics allows for a smooth transition from inflation to radiation domination, while the scalar quintessence-dark matter interaction avoids the possible dangerous kination regime that could last for 
too long a period. The model also allows for a proper transition from dark matter to dark energy, including an appropriate duration of the matter domination period and the transition to the accelerated regime toward today's epoch.

Interaction models, in general, have been studied also in the context of dynamical systems (see, for example, Refs. [55, 56, 60] and for a recent review, see Ref. [105]). Since analytical solutions can only be obtained in some very special and particular cases, a dynamical system analysis can allow one to obtain a qualitative picture of the problem, including the stability, scaling solutions and attractor points and also giving a qualitative view of the long-time behavior of the cosmological dynamics. In the present work we have adopted a more direct approach, by directly studying the background dynamics numerically. It would, however, be important to study the model presented here also in a dynamical system context. Here, we have performed an analysis for the predictions from per- turbations only for the cosmological inflationary regime. Extending such an analysis also for the late Universe dynamics (like computing the redshift-space distortion $f \sigma_{8}$ and a comparison with the analogous results from the $\Lambda$ CDM model), including also a statistical analysis, would certainly help to better constrain the parameters of the model. We expect to make these different explorations of the model in the future.

\section{ACKNOWLEDGMENTS}

G.B.F.L. is supported by a scholarship from Conselho Nacional de Desenvolvimento Científico e Tecnológico $(\mathrm{CNPq})$. R.O.R. is partially supported by research grants from CNPq, Grant No. 302545/2017-4, and Fundação Carlos Chagas Filho de Amparo à Pesquisa do Estado do Rio de Janeiro (FAPERJ), Grant No. E26/202.892/2017.
[1] N. Aghanim et al. [Planck Collaboration], Planck 2018 results. VI. Cosmological parameters, arXiv:1807.06209 [astro-ph.CO]. arXiv:1807.06209 2019

[2] L. Anderson et al. [BOSS Collaboration], The clustering of galaxies in the SDSS-III Baryon Oscillation Spectroscopic Survey: baryon acoustic oscillations in the Data Releases 10 and 11 Galaxy samples, Mon. Not. Roy. Astron. Soc. 441, no. 1, 24 (2014) doi:10.1093/mnras/stu523 arXiv:1312.4877 [astro-ph.CO]].

[3] E. A. Kazin et al., The WiggleZ Dark Energy Survey: improved distance measurements to $z=1$ with reconstruction of the baryonic acoustic feature, Mon. Not. Roy. Astron. Soc. 441, no. 4, 3524 (2014) doi:10.1093/mnras/stu778 arXiv:1401.0358 [astroph.CO]].

[4] J. E. Bautista et al., The SDSS-IV extended Baryon Oscillation Spectroscopic Survey: Baryon Acoustic Oscillations at redshift of 0.72 with the DR14 Luminous Red Galaxy Sample, Astrophys. J. 863, 110 (2018)

[5] T. M. C. Abbott et al. [DES Collaboration], Dark Energy Survey year 1 results: Cosmological constraints from galaxy clustering and weak lensing, Phys. Rev. D 98, no. 4, 043526 (2018)

[6] D. M. Scolnic et al., The Complete Light-curve Sample of Spectroscopically Confirmed SNe Ia from PanSTARRS1 and Cosmological Constraints from the Combined Pantheon Sample, Astrophys. J. 859, no. 2, 101 (2018)

[7] T. M. C. Abbott et al. [DES Collaboration], First Cosmology Results using Type Ia Supernovae from the Dark Energy Survey: Constraints on Cosmological Parameters, Astrophys. J. 872, no. 2, L30 (2019) doi:10.3847/2041-8213/ab04fa arXiv:1811.02374 [astroph.CO]].

[8] S. Capozziello, S. Nojiri and S. D. Odintsov, Unified phantom cosmology: Inflation, dark energy and dark matter under the same standard, Phys. Lett. B 632, 597 (2006) doi:10.1016/j.physletb.2005.11.012 hep-th/0507182.

[9] A. R. Liddle, C. Pahud and L. A. Urena-Lopez, Triple unification of inflation, dark matter, and dark energy using a single field, Phys. Rev. D 77, 121301 (2008) doi:10.1103/PhysRevD.77.121301 arXiv:0804.0869 [astro-ph]].

[10] A. B. Henriques, R. Potting and P. M. Sa, Unification of inflation, dark energy, and dark matter within the Salam-Sezgin cosmological model, Phys. Rev. D 79, 103522 (2009) doi:10.1103/PhysRevD.79.103522 arXiv:0903.2014 [astro-ph.CO]].

[11] N. Bose and A. S. Majumdar, Unified Model of $k$ Inflation, Dark Matter \& Dark Energy, Phys. Rev. D 80, 103508 (2009) doi:10.1103/PhysRevD.80.103508 arXiv:0907.2330 [astro-ph.CO]].

[12] J. De-Santiago and J. L. Cervantes-Cota, Generalizing a Unified Model of Dark Matter, Dark Energy, and Inflation with Non Canonical Kinetic Term, Phys. Rev. D 83, 063502 (2011) doi:10.1103/PhysRevD.83.063502 arXiv:1102.1777 [astro-ph.CO]].

[13] E. Guendelman, E. Nissimov and S. Pacheva, Quintessential Inflation, Unified Dark Energy and Dark Matter, and Higgs Mechanism, Bulg. J. Phys. 44, no. 1, 015 (2017) arXiv:1609.06915 [gr-qc]].

[14] E. J. Copeland, M. Sami and S. Tsujikawa, Dynamics of dark energy, Int. J. Mod. Phys. D 15, 1753 (2006) doi:10.1142/S021827180600942X hep-th/0603057.

[15] P. J. Steinhardt, L. M. Wang and I. Zlatev, Cosmological tracking solutions, Phys. Rev. D 59, 123504 (1999) doi:10.1103/PhysRevD.59.123504 astro-ph/9812313.

[16] P. J. E. Peebles and A. Vilenkin, Quintessential inflation, Phys. Rev. D 59, 063505 (1999) doi:10.1103/PhysRevD.59.063505 astro-ph/9810509.

[17] K. Dimopoulos and J. W. F. Valle, Modeling quintessential inflation, Astropart. Phys. 18, 287 (2002) doi:10.1016/S0927-6505(02)00115-9 astro-ph/0111417.

[18] R. Rosenfeld and J. A. Frieman, A Simple model for quintessential inflation, JCAP 0509, 003 (2005) doi:10.1088/1475-7516/2005/09/003 astro- 
$\mathrm{ph} / 0504191$.

[19] S. Nojiri and S. D. Odintsov, Unifying phantom inflation with late-time acceleration: Scalar phantomnon-phantom transition model and generalized holographic dark energy, Gen. Rel. Grav. 38, 1285 (2006) doi:10.1007/s10714-006-0301-6 hep-th/0506212.

[20] J. C. Bueno Sanchez and K. Dimopoulos, Trapped Quintessential Inflation, Phys. Lett. B 642, 294 (2006) Erratum: [Phys. Lett. B 647, $526 \quad$ (2007)] doi:10.1016/j.physletb.2006.09.045, 10.1016/j.physletb.2006.09.070 hep-th/0605258.

[21] R. Rosenfeld and J. A. Frieman, Cosmic microwave background and large-scale structure constraints on a simple quintessential inflation model, Phys. Rev. D 75 , 043513 (2007) doi:10.1103/PhysRevD.75.043513 astro$\mathrm{ph} / 0611241$.

[22] M. Bastero-Gil, A. Berera, B. M. Jackson and A. Taylor, Hybrid Quintessential Inflation, Phys. Lett. B 678, 157 (2009) doi:10.1016/j.physletb.2009.06.025 arXiv:0905.2937 [hep-ph]].

[23] Y. Ito, S. Nojiri and S. D. Odintsov, Stability of Accelerating Cosmology in Two Scalar-Tensor Theory: Little Rip versus de Sitter, Entropy 14, 1578 (2012) doi:10.3390/e14081578 arXiv:1111.5389 [hep-th]].

[24] K. Bamba, S. Capozziello, S. Nojiri and S. D. Odintsov, Dark energy cosmology: the equivalent description via different theoretical models and cosmography tests, Astrophys. Space Sci. 342, 155 (2012) doi:10.1007/s10509012-1181-8 arXiv:1205.3421 [gr-qc]].

[25] M. Wali Hossain, R. Myrzakulov, M. Sami and E. N. Saridakis, Unification of inflation and dark energy la quintessential inflation, Int. J. Mod. Phys. D 24, no. 05,1530014 (2015) doi:10.1142/S0218271815300141 arXiv:1410.6100 [gr-qc]].

[26] A. Berera, Warm inflation, Phys. Rev. Lett. 75, 3218 (1995) doi:10.1103/PhysRevLett.75.3218 astro$\mathrm{ph} / 9509049$.

[27] A. Berera, I. G. Moss and R. O. Ramos, Warm Inflation and its Microphysical Basis, Rept. Prog. Phys. 72, 026901 (2009) doi:10.1088/0034-4885/72/2/026901 arXiv:0808.1855 [hep-ph]].

[28] M. Bastero-Gil and A. Berera, Warm inflation model building, Int. J. Mod. Phys. A 24, 2207 (2009) doi:10.1142/S0217751X09044206 arXiv:0902.0521[hep$\mathrm{ph}]$.

[29] K. Dimopoulos and L. Donaldson-Wood, Warm quintessential inflation, Phys. Lett. B 796, 26 (2019) doi:10.1016/j.physletb.2019.07.017 arXiv:1906.09648 [gr-qc]].

[30] J. G. Rosa and L. B. Ventura, Warm Little Inflaton becomes Dark Energy, Phys. Lett. B 798, 134984 (2019) doi:10.1016/j.physletb.2019.134984 arXiv:1906.11835 [hep-ph]].

[31] A. Agarwal, R. Myrzakulov, M. Sami and N. K. Singh, Quintessential inflation in a thawing realization, Phys. Lett. B 770, 200 (2017) doi:10.1016/j.physletb.2017.04.066 arXiv:1708.00156 [gr-qc]].

[32] K. Dimopoulos, L. Donaldson Wood and C. Owen, Instant preheating in quintessential inflation with $\alpha$-attractors, Phys. Rev. D 97, no. 6, 063525 (2018) doi:10.1103/PhysRevD.97.063525 arXiv:1712.01760 [astro-ph.CO]].
[33] J. C. Bueno Sanchez and K. Dimopoulos, Curvaton reheating allows TeV Hubble scale in NO inflation, JCAP 0711, 007 (2007) doi:10.1088/1475-7516/2007/11/007 arXiv:0707.3967 [hep-ph]].

[34] T. Matsuda, NO Curvatons or Hybrid Quintessential Inflation, JCAP 0708, 003 (2007) doi:10.1088/14757516/2007/08/003 arXiv:0707.1948 [hep-ph]].

[35] T. Qiu and K. Feng, Reheating mechanism of the curvaton with nonminimal derivative coupling to gravity, Eur. Phys. J. C 77, no. 10, 687 (2017) doi:10.1140/epjc/s10052-017-5275-x arXiv:1608.01752 [hep-ph]].

[36] K. Dimopoulos and T. Markkanen, Non-minimal gravitational reheating during kination, JCAP $\mathbf{1 8 0 6}$, $021 \quad$ (2018) doi:10.1088/1475-7516/2018/06/021 arXiv:1803.07399 [gr-qc]].

[37] T. Opferkuch, P. Schwaller and B. A. Stefanek, Ricci Reheating, JCAP 1907, 016 (2019) doi:10.1088/14757516/2019/07/016 arXiv:1905.06823 [gr-qc]].

[38] K. Dimopoulos, M. Kariauskas and C. Owen, Quintessential inflation with a trap and axionic dark matter, Phys. Rev. D 100, no. 8, 083530 (2019) doi:10.1103/PhysRevD.100.083530 arXiv:1907.04676 [hep-ph]].

[39] E. Palti, The Swampland: Introduction and Review, Fortsch. Phys. 67, no. 6, 1900037 (2019) doi:10.1002/prop.201900037 arXiv:1903.06239 [hepth]].

[40] M. Motaharfar, V. Kamali and R. O. Ramos, Warm inflation as a way out of the swampland, Phys. Rev. D 99, no. 6, 063513 (2019) doi:10.1103/PhysRevD.99.063513 arXiv:1810.02816 [astro-ph.CO]].

[41] S. Das, Warm Inflation in the light of Swampland Criteria, Phys. Rev. D 99, no. 6, 063514 (2019) doi:10.1103/PhysRevD.99.063514 arXiv:1810.05038 [hep-th]].

[42] M. Bastero-Gil, A. Berera, R. O. Ramos and J. G. Rosa, Towards a reliable effective field theory of inflation, arXiv:1907.13410 [hep-ph].

[43] C. Q. Geng, M. W. Hossain, R. Myrzakulov, M. Sami and E. N. Saridakis, Quintessential inflation with canonical and noncanonical scalar fields and Planck 2015 results, Phys. Rev. D 92, no. 2, $023522(2015)$ doi:10.1103/PhysRevD.92.023522 arXiv:1502.03597 [gr-qc]].

[44] C. Q. Geng, C. C. Lee, M. Sami, E. N. Saridakis and A. A. Starobinsky, Observational constraints on successful model of quintessential Inflation, JCAP 1706, 011 (2017) doi:10.1088/1475-7516/2017/06/011 arXiv:1705.01329 [gr-qc]].

[45] S. Ahmad, R. Myrzakulov and M. Sami, Relic gravitational waves from Quintessential Inflation, Phys. Rev. D 96, no. 6, 063515 (2017) doi:10.1103/PhysRevD.96.063515 arXiv:1705.02133 [gr-qc]].

[46] M. Shahalam, W. Yang, R. Myrzakulov and A. Wang, Late-time acceleration with steep exponential potentials, Eur. Phys. J. C 77, no. 12, 894 (2017) doi:10.1140/epjc/s10052-017-5468-3 arXiv:1802.00326 [gr-qc]].

[47] S. Das, M. Banerjee and N. Roy, Dynamical System Analysis for Steep Potentials, JCAP 1908, 024 (2019) doi:10.1088/1475-7516/2019/08/024 arXiv:1903.02288 [gr-qc]]. 
[48] R. R. Caldwell and E. V. Linder, The Limits of quintessence, Phys. Rev. Lett. 95, 141301 (2005) doi:10.1103/PhysRevLett.95.141301 astro$\mathrm{ph} / 0505494$.

[49] S. Tsujikawa, Quintessence: A Review, Class. Quant. Grav. 30, 214003 (2013) doi:10.1088/02649381/30/21/214003 arXiv:1304.1961 [gr-qc]].

[50] T. Chiba, A. De Felice and S. Tsujikawa, Observational constraints on quintessence: thawing, tracker, and scaling models, Phys. Rev. D 87, no. 8, 083505 (2013) doi:10.1103/PhysRevD.87.083505 arXiv:1210.3859 [astro-ph.CO]].

[51] C. Wetterich, Growing neutrinos and cosmological selection, Phys. Lett. B 655, 201 (2007) doi:10.1016/j.physletb.2007.08.060 arXiv:0706.4427 [hep-ph]].

[52] L. Amendola, M. Baldi and C. Wetterich, Quintessence cosmologies with a growing matter component, Phys. Rev. D 78, 023015 (2008) doi:10.1103/PhysRevD.78.023015 arXiv:0706.3064 [astro-ph]].

[53] L. Amendola, Coupled quintessence, Phys. Rev. D 62, 043511 (2000) doi:10.1103/PhysRevD.62.043511 astro$\mathrm{ph} / 9908023$.

[54] S. del Campo, R. Herrera, G. Olivares and D. Pavon, Interacting models of soft coincidence, Phys. Rev. D 74, 023501 (2006) doi:10.1103/PhysRevD.74.023501 astro$\mathrm{ph} / 0606520$.

[55] L. Amendola, M. Quartin, S. Tsujikawa and I. Waga, Challenges for scaling cosmologies, Phys. Rev. D 74, 023525 (2006) doi:10.1103/PhysRevD.74.023525 astro$\mathrm{ph} / 0605488$.

[56] L. P. Chimento, M. I. Forte and G. M. Kremer, Cosmological model with interactions in the dark sector, Gen. Rel. Grav. 41, 1125 (2009) doi:10.1007/s10714008-0694-5 arXiv:0711.2646 [astro-ph]].

[57] R. Rosenfeld, Reconstruction of interacting dark energy models from parameterizations, Phys. Rev. D 75, 083509 (2007) doi:10.1103/PhysRevD.75.083509 astro$\mathrm{ph} / 0701213$.

[58] S. del Campo, R. Herrera and D. Pavon, Toward a solution of the coincidence problem, Phys. Rev. D 78, 021302 (2008) doi:10.1103/PhysRevD.78.021302 arXiv:0806.2116 [astro-ph]].

[59] S. del Campo, R. Herrera and D. Pavon, Interacting models may be key to solve the cosmic coincidence problem, JCAP 0901, 020 (2009) doi:10.1088/14757516/2009/01/020 arXiv:0812.2210 [gr-qc]].

[60] M. Quartin, M. O. Calvao, S. E. Joras, R. R. R. Reis and I. Waga, Dark Interactions and Cosmological FineTuning, JCAP 0805, 007 (2008) doi:10.1088/14757516/2008/05/007 arXiv:0802.0546 [astro-ph]].

[61] L. P. Chimento, Linear and nonlinear interactions in the dark sector, Phys. Rev. D 81, 043525 (2010) doi:10.1103/PhysRevD.81.043525 arXiv:0911.5687 [astro-ph.CO]].

[62] I. Duran, D. Pavon and W. Zimdahl, Observational constraints on a holographic, interacting dark energy model, JCAP 1007, 018 (2010) doi:10.1088/14757516/2010/07/018 arXiv:1007.0390 [astro-ph.CO]].

[63] F. Arevalo, A. P. R. Bacalhau and W. Zimdahl, Cosmological dynamics with non-linear interactions, Class. Quant. Grav. 29, 235001 (2012) doi:10.1088/02649381/29/23/235001 arXiv:1112.5095 [astro-ph.CO]].
[64] W. Zimdahl, Interactions in the dark sector of the Universe, Int. J. Geom. Meth. Mod. Phys. 11, 1460014 (2014) doi:10.1142/S0219887814600147 arXiv:1404.7334 [astro-ph.CO]].

[65] B. Y. Pu, X. D. Xu, B. Wang and E. Abdalla, Early dark energy and its interaction with dark matter, Phys. Rev. D 92, no. 12, 123537 (2015) doi:10.1103/PhysRevD.92.123537 arXiv:1412.4091 [astro-ph.CO]].

[66] E. G. M. Ferreira, J. Quintin, A. A. Costa, E. Abdalla and B. Wang, Evidence for interacting dark energy from BOSS, Phys. Rev. D 95, no. 4, 043520 (2017) doi:10.1103/PhysRevD.95.043520 arXiv:1412.2777 [astro-ph.CO]].

[67] M. Shahalam, S. D. Pathak, M. M. Verma, M. Y. Khlopov and R. Myrzakulov, Dynamics of interacting quintessence, Eur. Phys. J. C 75, no. 8, 395 (2015) doi:10.1140/epjc/s10052-015-3608-1 arXiv:1503.08712 [gr-qc]].

[68] L. Santos, W. Zhao, E. G. M. Ferreira and J. Quintin, Constraining interacting dark energy with $C M B$ and $B A O$ future surveys, Phys. Rev. D 96, no. 10, 103529 (2017) doi:10.1103/PhysRevD.96.103529 arXiv:1707.06827 [astro-ph.CO]].

[69] W. Yang, S. Pan, R. Herrera and S. Chakraborty, Large-scale (in) stability analysis of an exactly solved coupled dark-energy model, Phys. Rev. D 98, no. 4, 043517 (2018) doi:10.1103/PhysRevD.98.043517 arXiv:1808.01669 [gr-qc]].

[70] R. von Marttens, L. Casarini, D. F. Mota and W. Zimdahl, Cosmological constraints on parametrized interacting dark energy, Phys. Dark Univ. 23, 100248 (2019) doi:10.1016/j.dark.2018.10.007 arXiv:1807.11380 [astro-ph.CO]].

[71] M. Benetti, W. Miranda, H. A. Borges, C. Pigozzo, S. Carneiro and J. S. Alcaniz, Looking for interactions in the cosmological dark sector, arXiv:1908.07213 [astroph.CO].

[72] Y. L. Bolotin, A. Kostenko, O. A. Lemets and D. A. Yerokhin, Cosmological Evolution With Interaction Between Dark Energy And Dark Matter, Int. J. Mod. Phys. D 24, no. 03, 1530007 (2015) doi:10.1142/S0218271815300074 arXiv:1310.0085 [astro-ph.CO]].

[73] B. Wang, E. Abdalla, F. Atrio-Barandela and D. Pavon, Dark Matter and Dark Energy Interactions: Theoretical Challenges, Cosmological Implications and $\mathrm{Ob}$ servational Signatures, Rept. Prog. Phys. 79, no. 9, 096901 (2016) doi:10.1088/0034-4885/79/9/096901 arXiv:1603.08299 [astro-ph.CO]].

[74] M. Bastero-Gil, A. Berera and R. O. Ramos, Dissipation coefficients from scalar and fermion quantum field interactions, JCAP 1109, 033 (2011) doi:10.1088/14757516/2011/09/033 arXiv:1008.1929 [hep-ph]].

[75] M. Bastero-Gil, A. Berera, R. O. Ramos and J. G. Rosa, General dissipation coefficient in low-temperature warm inflation, JCAP 1301, 016 (2013) doi:10.1088/14757516/2013/01/016 arXiv:1207.0445 [hep-ph]].

[76] M. Bastero-Gil, A. Berera, R. O. Ramos and J. G. Rosa, Warm Little Inflaton, Phys. Rev. Lett. 117, no. 15, 151301 (2016) doi:10.1103/PhysRevLett.117.151301 arXiv:1604.08838 [hep-ph]].

[77] I. G. Moss and C. Xiong, On the consistency of warm inflation, JCAP 0811, 023 (2008) doi:10.1088/1475- 
7516/2008/11/023 arXiv:0808.0261 [astro-ph]].

[78] S. del Campo, R. Herrera, D. Pavón and J. R. Villanueva, On the consistency of warm inflation in the presence of viscosity, JCAP 1008, 002 (2010) doi:10.1088/1475-7516/2010/08/002 arXiv:1007.0103 [astro-ph.CO]].

[79] M. Bastero-Gil, A. Berera, R. Cerezo, R. O. Ramos and G. S. Vicente, Stability analysis for the background equations for inflation with dissipation and in a viscous radiation bath, JCAP 1211, 042 (2012) doi:10.1088/14757516/2012/11/042 arXiv:1209.0712 [astro-ph.CO]].

[80] M. Bastero-Gil, R. Cerezo and J. G. Rosa, Inflaton dark matter from incomplete decay, Phys. Rev. D 93, no. 10, 103531 (2016) doi:10.1103/PhysRevD.93.103531 arXiv:1501.05539 [hep-ph]].

[81] J. G. Rosa and L. B. Ventura, Warm Little Inflaton becomes Cold Dark Matter, Phys. Rev. Lett. 122, no. 16, 161301 (2019) doi:10.1103/PhysRevLett.122.161301 arXiv:1811.05493 [hep-ph]].

[82] I. Dymnikova and M. Khlopov, Self-consistent initial conditions in inflationary cosmology, Grav. Cosmol. Suppl. 4, 50 (1998). I. Dymnikova and M. Khlopov, Decay of cosmological constant as Bose condensate evaporation, Mod. Phys. Lett. A 15, 2305 (2000) doi:10.1142/S0217732300002966 astroph/0102094. I. Dymnikova and M. Khlopov, Decay of cosmological constant in selfconsistent inflation, Eur. Phys. J. C 20, 139 (2001). doi:10.1007/s100520100625

[83] R. H. Brandenberger and M. Yamaguchi, Spontaneous baryogenesis in warm inflation, Phys. Rev. D 68, 023505 (2003) doi:10.1103/PhysRevD.68.023505 hep$\mathrm{ph} / 0301270$.

[84] M. Bastero-Gil, A. Berera, R. O. Ramos and J. G. Rosa, Warm baryogenesis, Phys. Lett. B 712, 425 (2012) doi:10.1016/j.physletb.2012.05.032 arXiv:1110.3971 [hep-ph]].

[85] M. Bastero-Gil, A. Berera, R. O. Ramos and J. G. Rosa, Observational implications of mattergenesis during inflation, JCAP 1410, 053 (2014) doi:10.1088/14757516/2014/10/053 arXiv:1404.4976 [astro-ph.CO]].

[86] Y. Akrami et al. [Planck Collaboration], Planck 2018 results. X. Constraints on inflation, arXiv:1807.06211 [astro-ph.CO].

[87] D. Lyth and A. Liddle, The Primordial Density Perturbation: Cosmology, Inflation and the Origin of Structure (Cambridge University Press, Cambridge, 2009).

[88] R. O. Ramos and L. A. da Silva, Power spectrum for inflation models with quantum and thermal noises, JCAP 1303, 032 (2013) doi:10.1088/1475-7516/2013/03/032 arXiv:1302.3544 [astro-ph.CO]].

[89] S. Bartrum, M. Bastero-Gil, A. Berera, R. Cerezo, R. O. Ramos and J. G. Rosa, The importance of being warm (during inflation), Phys. Lett. B 732, 116 (2014) doi:10.1016/j.physletb.2014.03.029 arXiv:1307.5868 [hep-ph]].

[90] M. Benetti and R. O. Ramos, Warm inflation dissipative effects: predictions and constraints from the Planck data, Phys. Rev. D 95, no. 2, 023517 (2017) doi:10.1103/PhysRevD.95.023517 arXiv:1610.08758 [astro-ph.CO]].
[91] C. Graham and I. G. Moss, Density fluctuations from warm inflation, JCAP 0907, 013 (2009) doi:10.1088/1475-7516/2009/07/013 arXiv:0905.3500 [astro-ph.CO]].

[92] M. Bastero-Gil, A. Berera and R. O. Ramos, Shear viscous effects on the primordial power spectrum from warm inflation, JCAP 1107, 030 (2011) doi:10.1088/1475-7516/2011/07/030 arXiv:1106.0701 [astro-ph.CO]].

[93] M. Bastero-Gil, A. Berera, I. G. Moss and R. O. Ramos, Cosmological fluctuations of a random field and radiation fluid, JCAP 1405, 004 (2014) doi:10.1088/14757516/2014/05/004 arXiv:1401.1149 [astro-ph.CO]].

[94] V. Kamali, M. Motaharfar and R. O. Ramos, Warm brane inflation with an exponential potential: a consistent realization away from the swampland, arXiv:1910.06796 [gr-qc].

[95] M. Bastero-Gil, A. Berera, R. Brandenberger, I. G. Moss, R. O. Ramos and J. G. Rosa, The role of fluctuation-dissipation dynamics in setting initial conditions for inflation, JCAP 1801, 002 (2018) doi:10.1088/1475-7516/2018/01/002 arXiv:1612.04726 [astro-ph.CO]].

[96] A. R. Liddle and S. M. Leach, How long before the end of inflation were observable perturbations produced?, Phys. Rev. D 68, 103503 (2003) doi:10.1103/PhysRevD.68.103503 astro-ph/0305263.

[97] L. Amendola et al., Cosmology and fundamental physics with the Euclid satellite, Living Rev. Rel. 21, no. 1, 2 (2018) doi:10.1007/s41114-017-0010-3 arXiv:1606.00180 [astro-ph.CO]].

[98] W. Hu, R. Barkana and A. Gruzinov, Cold and fuzzy dark matter, Phys. Rev. Lett. 85, 1158 (2000) doi:10.1103/PhysRevLett.85.1158 astro-ph/0003365].

[99] P. A. R. Ade et al. [Planck Collaboration], Planck 2015 results. XIV. Dark energy and modified gravity, Astron. Astrophys. 594, A14 (2016) doi:10.1051/00046361/201525814 arXiv:1502.01590 [astro-ph.CO]].

[100] J. F. Jesus, R. Valentim, A. A. Escobal and S. H. Pereira, Gaussian Process Estimation of Transition Redshift, arXiv:1909.00090 [astro-ph.CO].

[101] V. Sahni, M. Sami and T. Souradeep, Relic gravity waves from brane world inflation, Phys. Rev. D 65, 023518 (2001) doi:10.1103/PhysRevD.65.023518 grqc/0105121.

[102] A. Hosoya and M. a. Sakagami, Time Development of Higgs Field at Finite Temperature, Phys. Rev. D 29, 2228 (1984). doi:10.1103/PhysRevD.29.2228

[103] M. Morikawa and M. Sasaki, Entropy Production in the Inflationary Universe, Prog. Theor. Phys. 72, 782 (1984). doi:10.1143/PTP.72.782

[104] I. G. Moss and C. M. Graham, Particle production and reheating in the inflationary universe, Phys. Rev. D 78, 123526 (2008) doi:10.1103/PhysRevD.78.123526 arXiv:0810.2039 [hep-ph]].

[105] S. Bahamonde, C. G. Bhmer, S. Carloni, E. J. Copeland, W. Fang and N. Tamanini, Dynamical systems applied to cosmology: dark energy and modified gravity, Phys. Rept. 775-777, 1 (2018) doi:10.1016/j.physrep.2018.09.001 arXiv:1712.03107 [gr-qc]]. 